\title{
MODELADO Y CONTROL DE UNA PLANTA PILOTO DE NANOFILTRACIÓN APLICADA A LA REUTILIZACIÓN DE AGUAS RESIDUALES EN AGRICULTURA
}

\author{
A. I. Sánchez-Pelegrina ${ }^{1}$, F. Rodríguez ${ }^{1 *}$, I. Oller ${ }^{2}$, M. Berenguel ${ }^{1}$, S. Malato ${ }^{2}$ \\ ${ }^{1}$ Departamento de Informática. Universidad de Almería, CIESOL, CeiA3. Almería, *frrodrig@ual.es \\ ${ }^{2}$ Unidad de Tratamientos Solares de Agua, Plataforma Solar de Almería; CIEMAT
}

\begin{abstract}
Resumen
Actualmente es necesario el uso de instalaciones de tratamiento de aguas residuales debido a la escasez de recursos hídricos de calidad. Las plantas de nanofiltración presentan cada vez más interés, ya que requieren menos energía que otros sistemas. En la Plataforma Solar de Almería se utiliza una planta de nanofiltración gestionada de forma manual basándose en la experiencia de los operarios. En este trabajo se muestra el proceso de análisis, modelado y control de esta planta, que ha ayudado a mejorar el conocimiento de la misma, a determinar su punto de operación e identificar las variables que más influyen en el comportamiento del sistema. Además, con la arquitectura de control propuesta, basada en un controlador multivariable con desacoplo, se puede llegar a alcanzar unas condiciones idóneas de funcionamiento en cuanto a producción, seguridad y mantenimiento de las membranas, como se puede observar en los resultados que se presentan.
\end{abstract}

Palabras clave: Control multivariable, tratamiento de aguas contaminadas, modelo dinámico

\section{INTRODUCCIÓN}

La disponibilidad del recurso hídrico se ha reducido de forma considerable en las últimas décadas. A esta falta de agua se añade su baja calidad, debido fundamentalmente a la incorporación de trazas de plaguicidas y otras sustancias derivadas de la actividad humana [3]. Por este motivo se implantó una legislación sobre aguas a nivel europeo, con la adopción de la directiva marco sobre el agua, DMA(200/60/CE), que establece la gestión y protección de las aguas tanto superficiales como subterráneas, a fin de proveer una buena calidad y poder reducir y prevenir su contaminación [3].

Respondiendo a esta mala calidad de agua y a la necesidad de mantener los contaminantes en concentraciones adecuadas, se han desarrollado tratamientos terciarios, que se clasifican dependiendo de su fuerza impulsora y del tamaño de partícula que sea necesario tratar: microfiltración, ultrafiltración, ósmosis inversa o nanofiltración, electrodiálisis o adsorción. Concretamente, este trabajo se centra en una planta de nanofiltración capaz de separar sustancias orgánicas a menores presiones que otros procesos, por lo que es económicamente más viable al requerir menos energía que otros tratamientos [6]. Sin embargo, presenta como desventaja el ensuciamiento de la membrana, pues crea una resistencia adicional al paso del líquido, al reducirse el tamaño de poro, llegando a un límite donde hay que suspender la producción para someter a la planta a procesos de mantenimiento, como limpieza o sustitución de las membranas [4].

La planta objeto de estudio en este trabajo se ha estado utilizando hasta el momento de forma manual y se desconocían sus márgenes de regulación, su punto de operación óptimo y los rangos de operatividad de la instrumentación [4]. Para poder mejorar la productividad y evitar la dependencia de un operador en planta, se planteó el diseño de un sistema de control de las variables principales del sistema cuyos resultados se presentan en este artículo. Existen distintos trabajos para plantas similares como las de ósmosis inversa [1], pero para plantas con nanomembranas sólo destaca el desarrollo Stoller y Mendes basado en PIDs adaptativos [5] utilizando un sistema diferente a la planta piloto objeto de estudio.

En este artículo se describirá la planta piloto, su modelado, el diseño de un sistema de control multivariable y se mostrarán resultados preliminares obtenidos en ensayos reales.

\section{DESCRIPCIÓN DE LA PLANTA}

La planta piloto de nanofiltración (figura 1) se encuentra situada en la Plataforma Solar de Almería (CIEMAT), y su funcionamiento se describe detalladamente en [4]. 


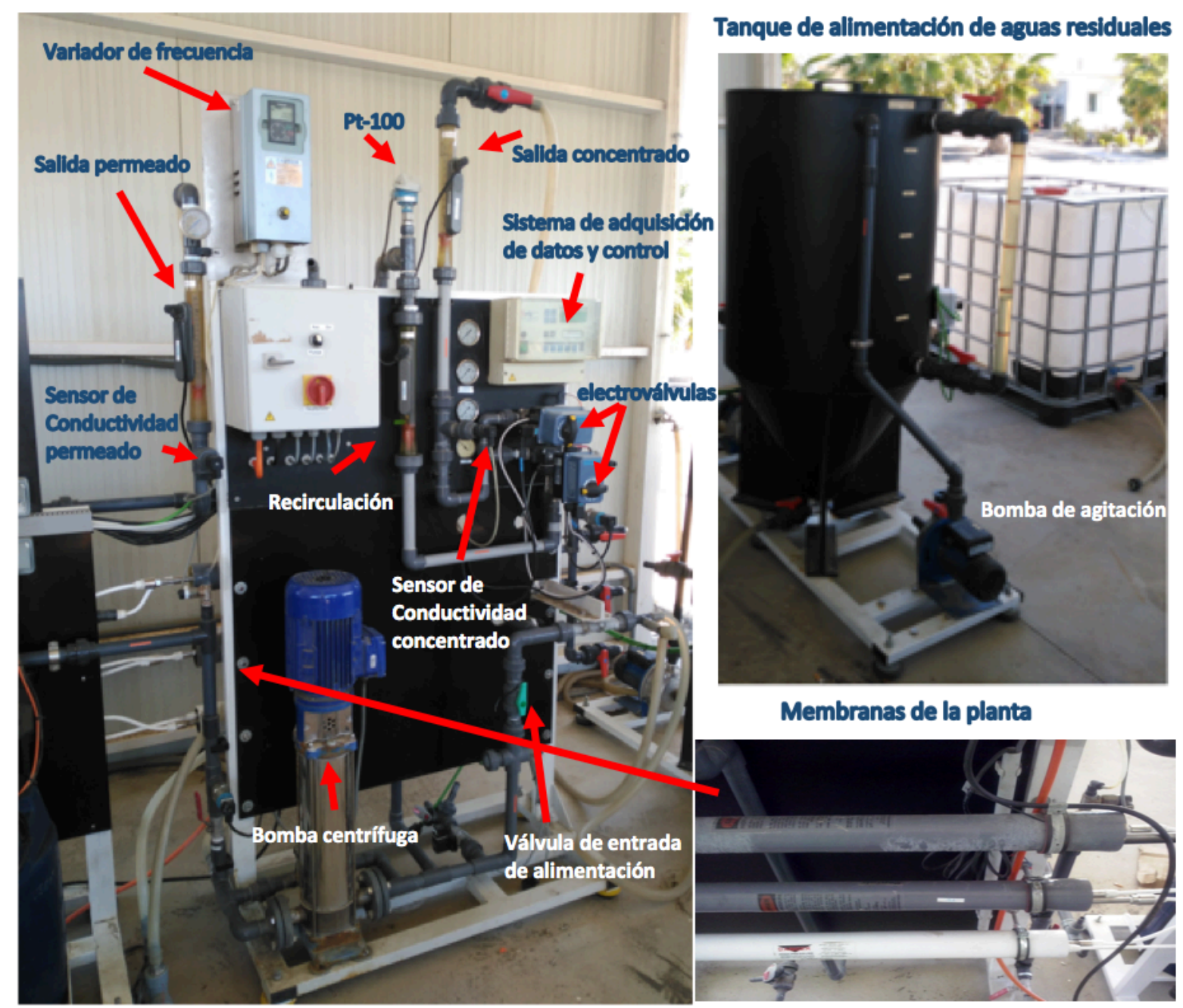

Figura 1.a. Elementos de la planta piloto

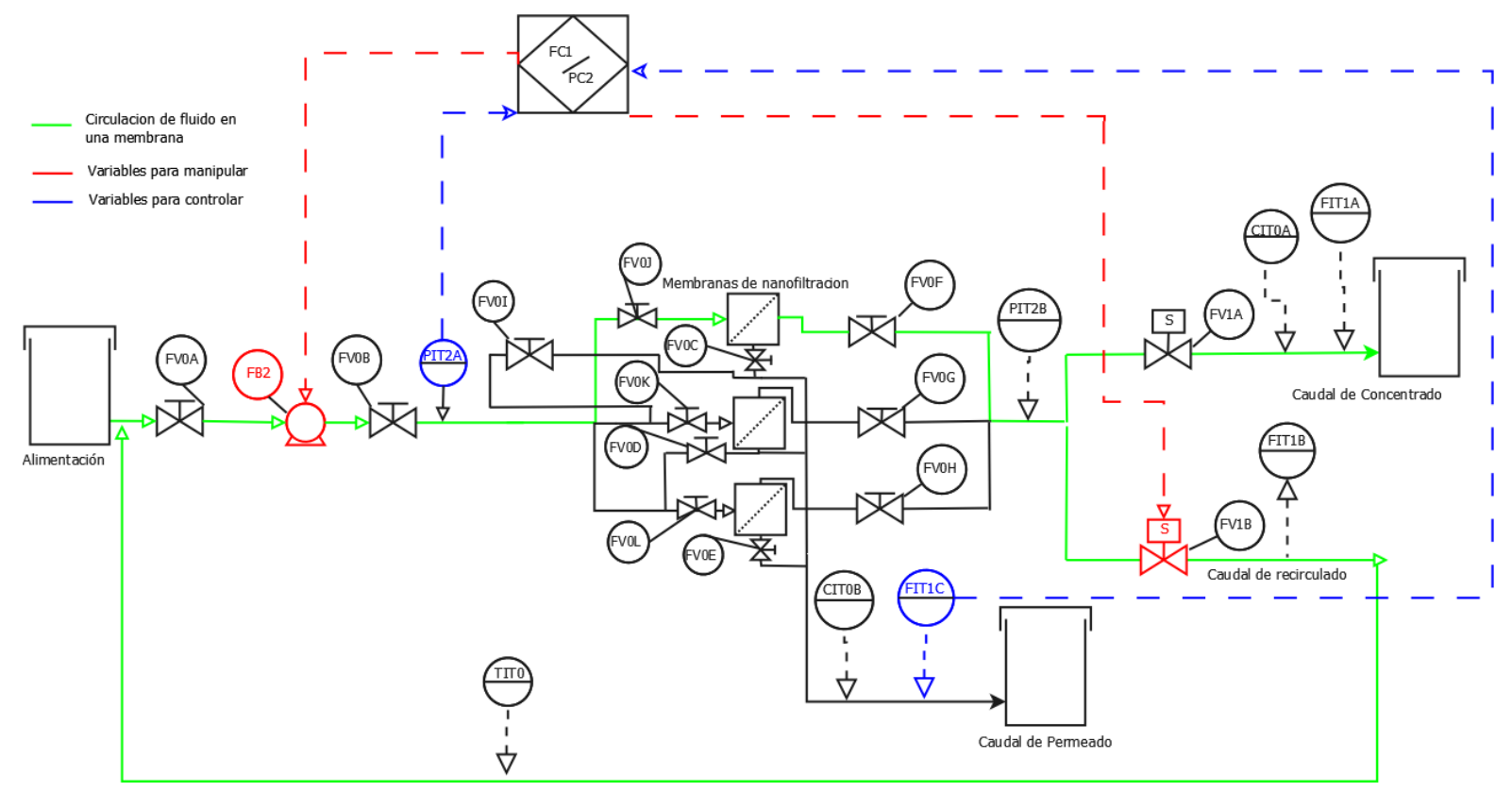

Figura 1.b. Instrumentación de la planta piloto

Figura 1: Planta piloto de nanofiltración 
Como se puede observar en la figura 1.b, la planta dispone de tres membranas de nanofiltración que se pueden utilizar en paralelo o en serie según la posición de la válvula FV0I, utilizándose esta última configuración en este trabajo. $\mathrm{Su}$ funcionamiento consiste en que la fuente de agua residual (que se ha simulado mediante el tanque de alimentación) se introduce en el sistema, que mantiene un determinado caudal mediante la bomba FB2, dividiéndose en tres ramas o líneas:

- Permeado, correspondiente al agua ya tratada y de calidad para determinados procesos.

- Concentrado, correspondiente al agua rechazada por su alta concentración de contaminantes.

- Recirculado que se utiliza para regular la presión transmembrana (mediante la válvula FV1B) con la que es necesario trabajar y que no podrá ser superior a 6 bares para evitar rotura de las tuberías y las membranas.

Tal y como está configurada la planta se podría controlar el caudal y la conductividad eléctrica (CE) de cada rama, así como la presión transmembrana, mediante la bomba $\mathrm{FB} 2$, la válvula de recirculado FV1B y la de concentrado FV1A. En una primera aproximación se ha decidido controlar el caudal de permeado y la presión de entrada en las membranas, considerando la conductividad eléctrica del agua residual de entrada como una perturbación (véase figura 2), descartando la idea de controlarla al no disponer de un elemento de medida que registre la conductividad a la entrada del sistema y al no ser una variable tan fundamental como las otras en los objetivos de la instalación.

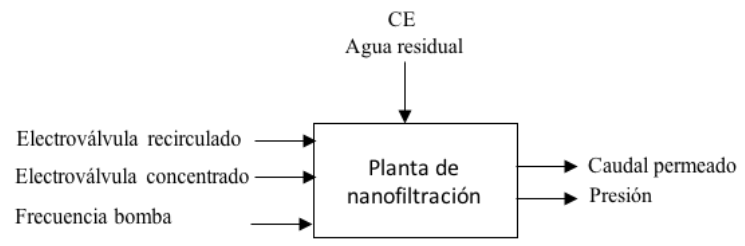

Figura 2: Diagrama de Entradas/Salidas de la planta

En el anexo 1 se muestra la instrumentación instalada en la planta. Para supervisar el proceso, la manipulación y la obtención de los parámetros, se diseñó un sistema supervisor de control y adquisición de datos (SCADA) que se desarrolló junto con el Departamento de Instrumentación de la Plataforma Solar de Almería.

\section{MODELADO DE LA PLANTA}

\subsection{DETERMINACIÓN DEL PUNTO DE OPERACIÓN}

Debido a que no se dispone de modelos matemáticos de la planta y de sus subsistemas (principalmente de las membranas, cuyo modelado es extremadamente complejo), para poder obtener su punto de operación óptimo en base a un determinado criterio (ya sea caudal o calidad), se procedió a la determinación de un punto idóneo de operación mediante los siguientes ensayos en bucle abierto en los que se modificaba el estado de las variables de control y de las perturbaciones:

- Apertura de válvula de recirculado (FV1B) manteniendo constante la válvula de concentrado y la frecuencia de trabajo de la bomba.

- Apertura de válvula de concentrado (FV1A) manteniendo constante la válvula de recirculado y la frecuencia de trabajo de la bomba.

- Variación de la frecuencia de la bomba (FB2) manteniendo constante la apertura de las dos válvulas.

- Repetición de los experimentos anteriores con diferentes conductividades del agua de entrada desde agua destilada hasta $6300[\mu \mathrm{S} / \mathrm{cm}]$ de cloruro sódico $(\mathrm{NaCl})$.

Como ejemplo, en la tabla 1 se muestran los valores obtenidos en estado estacionario para diferentes configuraciones de las entradas de control con una $\mathrm{CE}$ de $2300[\mu \mathrm{S} / \mathrm{cm}]$.

Tabla 1. Comportamiento estático de la planta

\begin{tabular}{|c|c|c|c|c|c|c|}
\hline $\begin{array}{c}\text { FB2 } \\
{[\mathbf{H z}]}\end{array}$ & $\begin{array}{c}\text { FV1B } \\
{[\%]}\end{array}$ & $\begin{array}{c}\text { FV1A } \\
{[\%]}\end{array}$ & $\begin{array}{c}\text { FIT1C } \\
{[\mathbf{L} / \mathbf{h}]}\end{array}$ & $\begin{array}{c}\text { FIT1A } \\
{[\mathbf{L} / \mathbf{h}]}\end{array}$ & $\begin{array}{c}\text { FIT1B } \\
{[\mathbf{L} / \mathbf{h}]}\end{array}$ & $\begin{array}{c}\text { PIT2A } \\
{[\mathbf{b a r}]}\end{array}$ \\
\hline \multirow{2}{*}{25} & 17 & 28 & 26,74 & 22 & 619,1 & 2,94 \\
\cline { 2 - 7 } & 26 & 39 & 19,08 & 118 & 754,8 & 2,5 \\
\hline \multirow{2}{*}{27} & 18 & 26 & 32,42 & 24,8 & 598,3 & 3,43 \\
\cline { 2 - 7 } & 24 & 40 & 15,49 & 118 & 788,4 & 2,87 \\
\hline \multirow{2}{*}{30} & 17 & 26 & 43,38 & 26,1 & 622,6 & 4,38 \\
\cline { 2 - 7 } & 35 & 48 & 26,91 & 116,6 & 979,9 & 3,49 \\
\hline \multirow{2}{*}{32,2} & 18 & 26 & 51,96 & 26,3 & 648,5 & 4,94 \\
\cline { 2 - 7 } & 29 & 50 & 30,59 & 118 & 1052,9 & 3,96 \\
\hline \multirow{2}{*}{34,7} & 17 & 24 & 60,48 & 23,2 & 702,8 & 5,54 \\
\cline { 2 - 7 } & 27 & 50 & 34,59 & 118 & 1153,2 & 4,47 \\
\hline
\end{tabular}

A medida que se fueron realizando los ensayos, se observaron los siguientes comportamientos:

- A medida que se incrementa la frecuencia de la bomba, se produce un incremento en la presión transmembrana y en el caudal de permeado. Además, se produce un aumento en el rango de apertura máximo de las válvulas.

- A mayor apertura de las válvulas, menor presión se genera, aumentándose el caudal de concentrado y recirculado y disminuyendo significativamente el caudal de permeado.

- La válvula de concentrado presenta problemas de saturación con un margen de regulación bajo, por lo que se mantiene con una apertura constante.

- La variación en la CE del agua entrada influye sobre el caudal de permeado de forma exponencial, pero no se observa efecto sobre la presión de entrada de las membranas. 
Finalmente, se seleccionó el punto de operación que permitiera la máxima productividad y no pusiera en riesgo el sistema, correspondiente a un caudal de permeado de $35,5[\mathrm{~L} / \mathrm{h}]$ y una presión de entrada de la membrana de 4,3 [bar]. Para una CE de 2300 $[\mu \mathrm{S} / \mathrm{cm}]$, la frecuencia de la bomba corresponde a unos 30 [Hz], la válvula de concentrado a 18 [\%] y la de recirculado en torno a 26 [\%].

\subsection{MODELADO DE LA PLANTA}

Una vez determinado el punto de operación, se procedió a realizar ensayos de curva de reacción con la misma metodología descrita en la sección anterior, observándose comportamientos de las variables a controlar similares a los de un sistema de primer orden con retardo tanto frente a las variables de control como a la variable de perturbación, tal y como se puede observar en la figura 3 , que como ejemplo, muestra el comportamiento del caudal de permeado y la presión frente a la válvula de recirculado junto con la respuesta del modelo obtenido. Los tiempos de retardo se han redondeado como múltiplos del periodo de muestreo del sistema de adquisición de datos (1 s). La figura 4 muestra la respuesta del caudal del permeado ante un cambio en la CE del agua de alimentación.
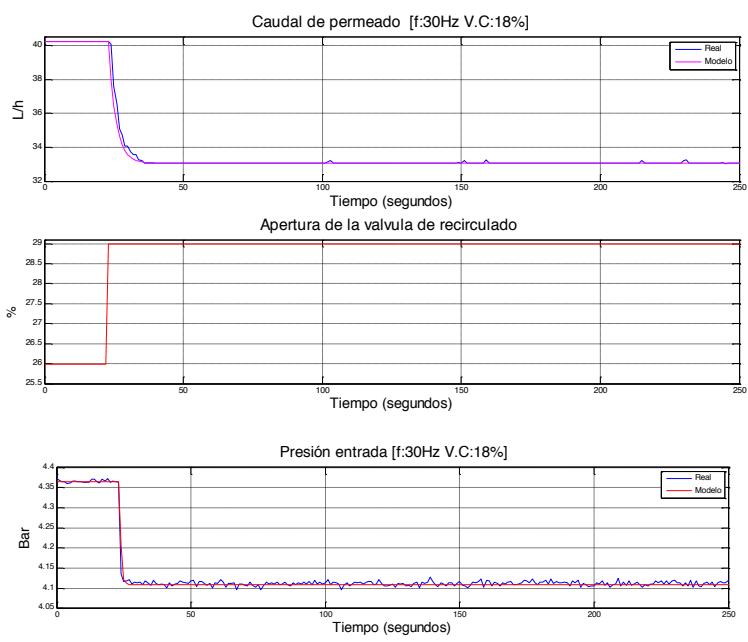

Figura 3: Respuesta del caudal de permeado y la presión frente a la apertura de la válvula.

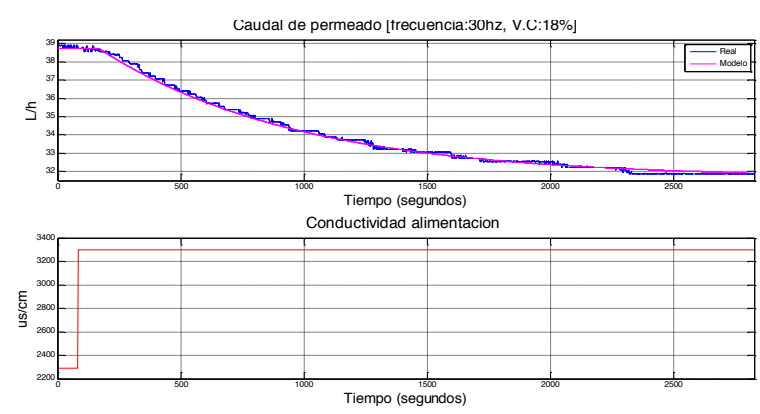

Figura 4: Respuesta del caudal de permeado frente a un cambio de la CE del agua de alimentación.
Expresado mediante la matriz de funciones de transferencia, el modelado de la planta viene dado por la siguiente descripción:

$\left[\begin{array}{l}Y_{1}(s) \\ Y_{2}(s)\end{array}\right]=\left[\begin{array}{ll}G_{11}(s) & G_{12}(s) \\ G_{21}(s) & G_{22}(s)\end{array}\right] \cdot\left[\begin{array}{l}U_{1}(s) \\ U_{2}(s)\end{array}\right]+\left[\begin{array}{l}P_{13}(s) \\ P_{23}(s)\end{array}\right] \cdot\left[U_{3}(s)\right]$

donde $U(s)$ corresponde a las variables de entrada:

- $U_{1}(s)$ : Frecuencia de la bomba [\%]

- $U_{2}(s)$ : Válvula de recirculado [\%]

- $U_{3}(s)$ : Conductividad en la alimentación $[\mu \mathrm{s} / \mathrm{cm}]$

$Y(s)$ corresponde a las variables de salida, que son:

- $Y_{1}(s)$ : Presión de entrada en la membrana [bar]

- $Y_{2}(s)$ : Caudal de permeado [L/h]

$G_{n m}(s)$ son las funciones de transferencia de las variables de control y $P_{n m}(s)$ las funciones de transferencia con respecto a la perturbación; todas ellas definidas de forma general según la ecuación (2) y cuyos parámetros se especifican en la Tabla 2.

$$
G_{n m}(s)=\frac{k_{n m}}{1+s \cdot \tau_{n m}} \cdot e^{-t r_{n m} \cdot s}
$$

Tabla 2. Características dinámicas del sistema

\begin{tabular}{|c|c|c|c|c|}
\hline$G_{n m}(s)$ & \multicolumn{2}{|c|}{$\mathrm{k}_{\mathrm{nm}}$} & $\begin{array}{c}\tau_{n m} \\
{[\mathrm{seg}]}\end{array}$ & $\begin{array}{r}\mathrm{tr}_{\mathrm{nm}} \\
\text { [seg] }\end{array}$ \\
\hline$G_{11}(s)$ & 0,085 & {$\left[\frac{b a r}{\%_{h z}}\right]$} & 1 & 0 \\
\hline$G_{12}(s)$ & $-0,06804$ & {$\left[\frac{b a r}{\%_{R}}\right]$} & 3,189 & 0 \\
\hline$G_{21}(s)$ & 0,6895 & {$\left[\frac{L_{p}}{h \cdot \%_{h z}}\right]$} & 0,878 & 0 \\
\hline$G_{22}(s)$ & $-1,78$ & {$\left[\frac{L_{p}}{h \cdot \%_{R}}\right]$} & 3,725 & 1 \\
\hline$P_{13}(s)$ & 0 & {$\left[\frac{\mathrm{bar} \cdot \mathrm{cm}}{\mu \cdot s}\right]$} & 0 & 0 \\
\hline$P_{23}(s)$ & $-0,00791$ & $\left\lceil\frac{L_{p} \cdot c m}{h \cdot \mu \cdot s}\right]$ & 813,42 & 50 \\
\hline
\end{tabular}

\section{CONTROL MULTIVARIABLE}

En base a la matriz de funciones de transferencia se determinó que la matriz de ganancias estáticas es la siguiente:

$$
\begin{gathered}
k=G(0)=\lim _{s \rightarrow 0} G(s)=\left(\begin{array}{ll}
k_{11} & k_{12} \\
k_{21} & k_{22}
\end{array}\right) \\
k=\left[\begin{array}{cc}
0,085 & -0,06804 \\
0,6895 & -1,78
\end{array}\right]
\end{gathered}
$$

así como que el índice de interacción de Bristol

$$
\lambda=\frac{1}{1-\frac{k_{12} \cdot k_{21}}{k_{11} \cdot k_{22}}}=1,4494
$$


resultando la matriz RGA [2] de la ecuación (5):

$\Lambda=\left[\begin{array}{cc}\lambda_{11} & 1-\lambda_{11} \\ 1-\lambda_{11} & \lambda_{11}\end{array}\right]=\left[\begin{array}{cc}1,4494 & -0,4494 \\ -0,4494 & 1,4494\end{array}\right](5)$

Del análisis de estos resultados se pueden definir las siguientes reglas de emparejamiento (que por otro lado parece lógicas):

- La presión a la entrada se empareja con la frecuencia de la bomba.

- El caudal de permeado se empareja con la válvula de recirculado.

En base a estos resultados, el diseño del controlador consta de dos lazos de control, $\boldsymbol{C}_{\mathbf{1}}(\boldsymbol{s})$ y $\boldsymbol{C}_{\mathbf{2}}(\boldsymbol{s})$, tipo PI con antiwindup [2] tal y como se puede observar en la figura 5, donde las funciones de transferencia $\boldsymbol{G}_{\boldsymbol{n} \boldsymbol{m}}(\boldsymbol{s})$ son los elementos de la matriz $2 \times 2$ que multiplica a las entradas de control en la ecuación (1) y $\boldsymbol{P}_{23}(\boldsymbol{s})$ la que describe el efecto de la perturbación, CE de la alimentación, entrada $\boldsymbol{u}_{3}(\boldsymbol{s})$. Además, en el segundo lazo se introdujo un desacoplador para corregir las interacciones producidas por el lazo uno que se observaron en la práctica, como se comenta más adelante en esta sección. Hay que indicar que, en su implementación en planta, se incluyó un conmutador Manual-Automático, estableciendo las conexiones adecuadas, en modo seguimiento mediante un mecanismo de transferencia sin saltos, para evitar cambios bruscos al realizar la conmutación.

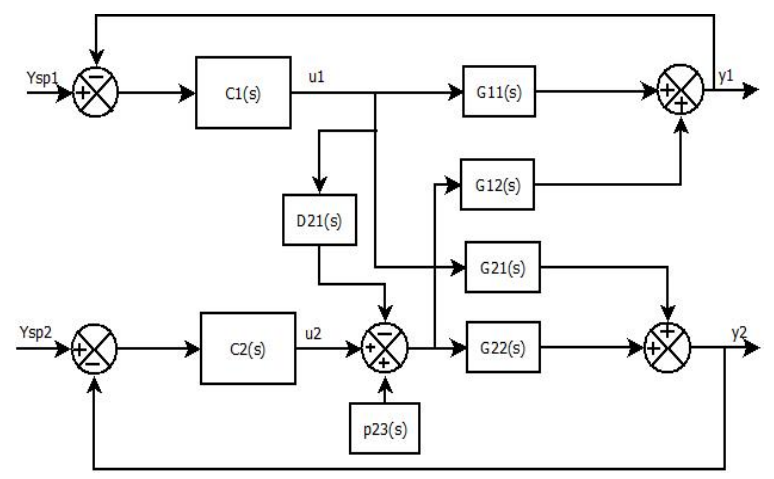

Figura 5: Esquema de control multivariable propuesto $\left(\boldsymbol{Y}_{\boldsymbol{s p i}}\right.$ son las referencias a los lazos de control)

En base a las especificaciones de diseño y los modelos obtenidos se diseñaron los siguientes controladores PI (incluyendo anti-windup):

- Controlador de presión. Se trata de un sistema de primer orden sin retardo, y se deseaba una respuesta en lazo cerrado de primer orden con una constante de tiempo igual o menor que la del sistema a controlar frente al seguimiento de referencias, por lo que se utilizó el método de cancelación polo-cero, resultando:

$$
C_{1}(s)=13,841 \cdot\left(1+\frac{1}{s}\right)
$$

- Controlador de caudal. Se trata de un sistema de primer orden con retardo, y se deseaba una respuesta amortiguada frente al seguimiento de referencias y el rechazo de perturbaciones, por lo que se utilizó el método Amigo [2], resultando:

$$
C_{2}(s)=-0,287 \cdot\left(1+\frac{1}{3,324 \cdot s}\right)
$$

En simulación se comprobó que los controladores diseñados cumplían las especificaciones, pero se observó que, al modificar la consigna de la presión de entrada, tenía un efecto más acentuado sobre el caudal de permeado que el que se había previsto por el resultado del RGA, por lo que se decidió incluir un desacoplador, $D_{21}(s)$ para atenuar los efectos, tal y como se puede observar en la figura 6 .

En el caso de modificar la consigna en el caudal de permeado no fue necesario utilizar un desacoplador ya que la interacción no era significativa.

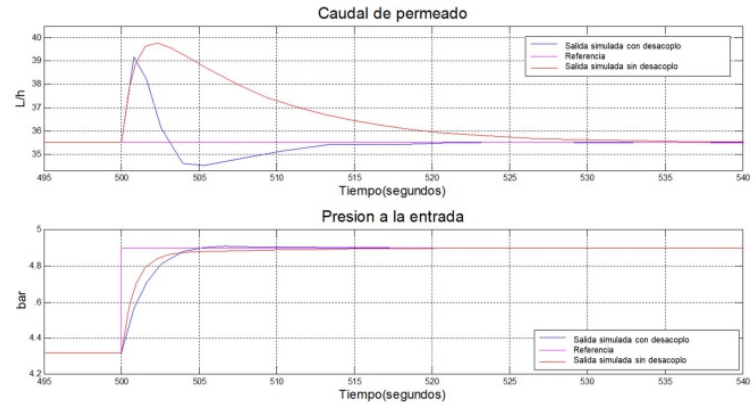

Figura 6: Efecto sobre el caudal de un cambio de consigna en la presión son y sin desacoplo.

Se realizaron ensayos en la planta para controlar el caudal de permeado y la presión a la entrada de la membrana, en torno a un punto de operación establecido, trabajando con las membranas en serie, y usando como entrada patrón agua con cloruro sódico a una conductividad de $2300 \mu \mathrm{s} / \mathrm{cm}$. Como ejemplo de resultados preliminares, la figura 7 muestra resultados en la planta real de la regulación de las variables de salida sin desacoplo y, en la figura 8 se puede observar el comportamiento con la implementación del desacoplo.

Por problemas de disponibilidad de la instalación (que es utilizada para distintos propósitos), sólo se han podido realizar estos ensayos para analizar si el sistema alcanza las referencias establecidas y si rechaza las perturbaciones que se comentan a continuación, quedando pendientes ensayos con cambios en las referencias a lo largo de la operación (en los ensayos mostrados sólo hay un cambio de consigna al principio, para llevar al sistema al punto de operación). Hay que hacer notar que se trata de un circuito hidráulico con tres derivaciones (permeado, concentrado y recirculado), que interaccionan entre sí. Aunque una de las válvulas tiene apertura constante, cualquier cambio en la presión 
intramembrana afecta a los caudales. En las gráficas se puede observar que hay dos fuentes principales de perturbación en los ensayos (además de la ya contemplada de CE). Por un lado, debido a la naturaleza de los sensores de caudal utilizados (rotámetros), estos se ven afectados por diferencias de presión (por pequeñas que sean), además de por variaciones de temperatura y de CE. Por tanto, aparecen de forma irregular picos en la respuesta que afectan al sistema de control, que intenta mitigar su efecto (ver figuras 7 y 8). Aunque se intente filtrar la señal de medida, no se pueden evitar esos picos porque para hacerlo se afectaría a la dinámica fundamental del sistema. Es un problema que hay que estudiar con un mayor detalle para ensayos futuros. Obviamente, el caudal de permeado y la presión están estrechamente relacionadas (p.e. a más presión, mayor caudal de permeado se obtiene).
La hipótesis que manejan los autores a la vista de los ensayos es que cuando la presión disminuye (como ocurre en la figura 7), suele deberse a una entrada de aire en el sistema; por otra parte, cuando hay un aumento de presión (también en figura 7), se debe a un cambio en la salinidad, produciéndose un taponamiento transitorio de los poros de las membranas, que tardan poco en liberarse debido a que el tamaño de partícula del cloruro sódico es menor que el de los poros de la membrana, volviendo a la situación nominal. Se puede observar también que la frecuencia de la bomba presenta una nolinealidad de tipo "cuantificador". En las gráficas aparece el ruido inherente a la medida de presión. La sintonía utilizada para el controlador de la válvula de recirculado produce respuestas conservadoras que habrá que mejorar en futuros ensayos.
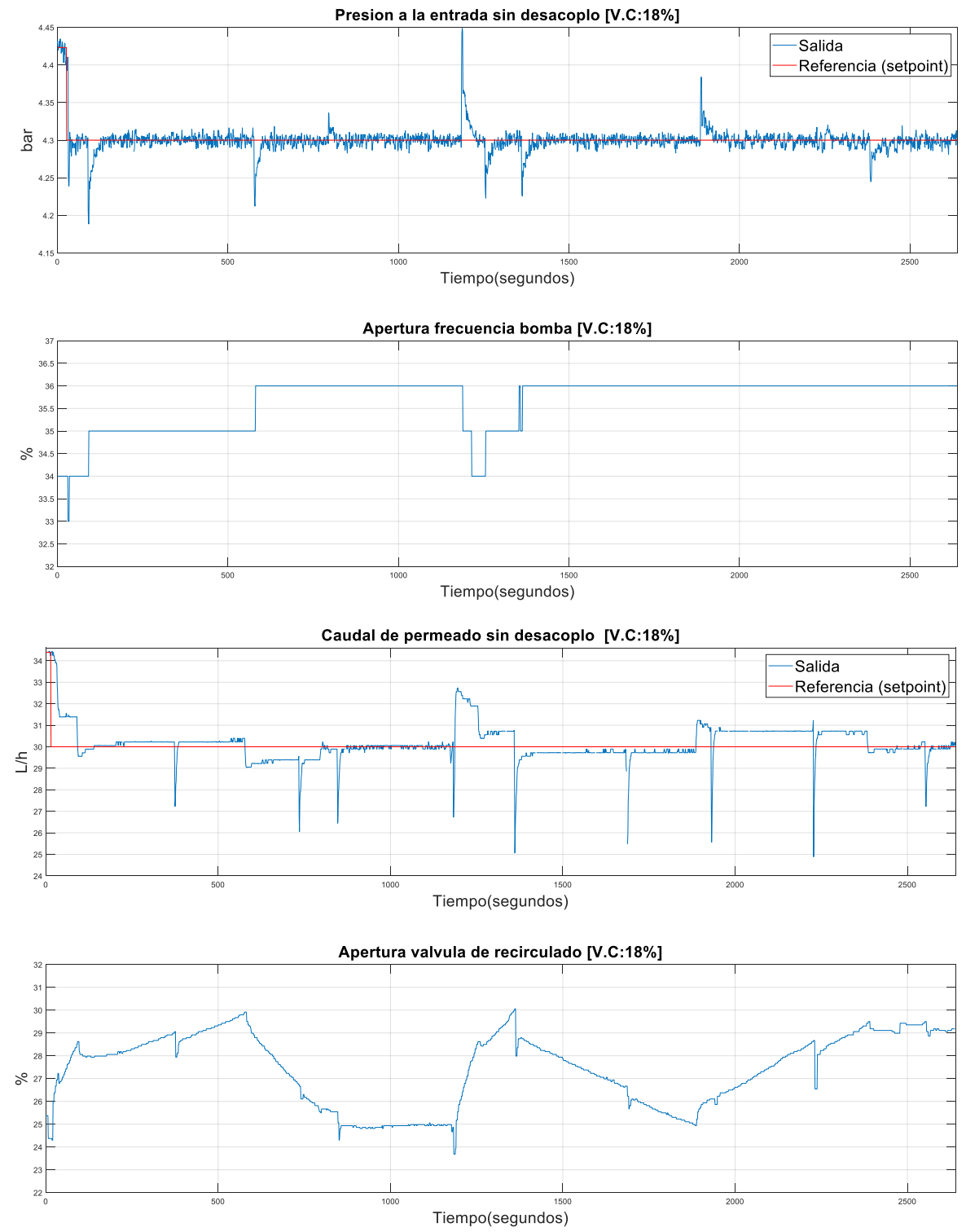

Figura 7: Comportamiento de presión y caudal sin desacoplo. 

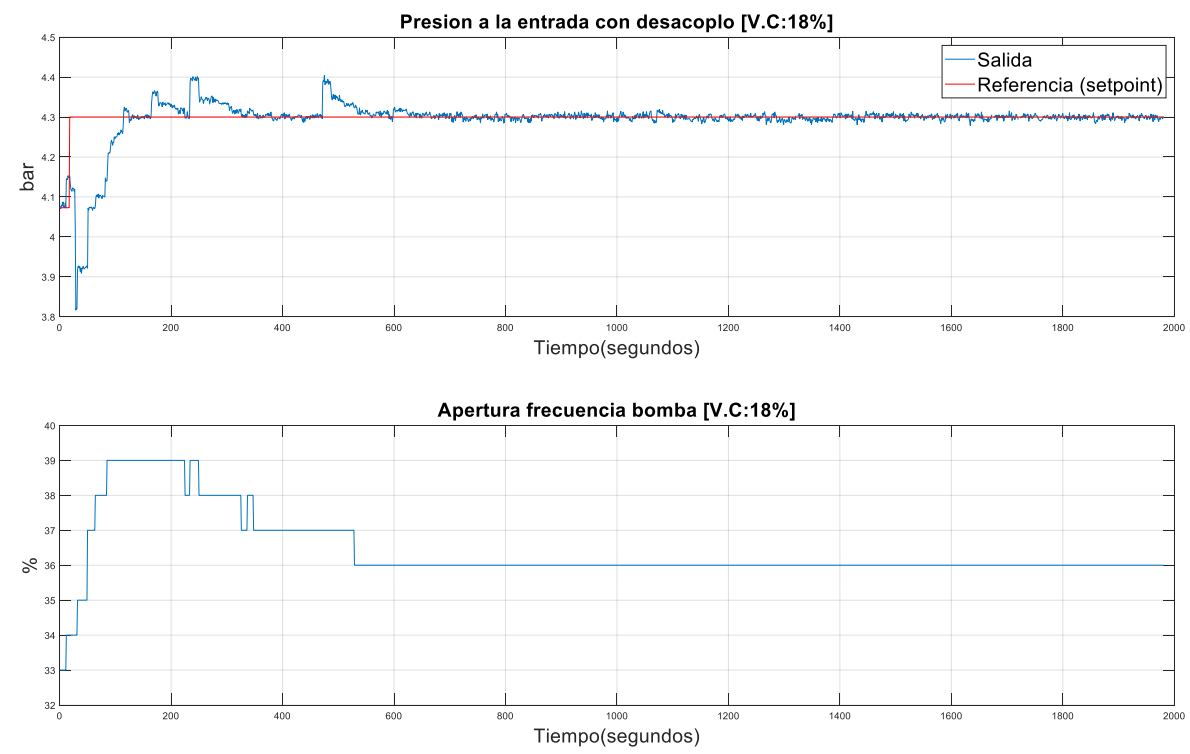

Figura 8: Comportamiento de presión y caudal con desacoplo.
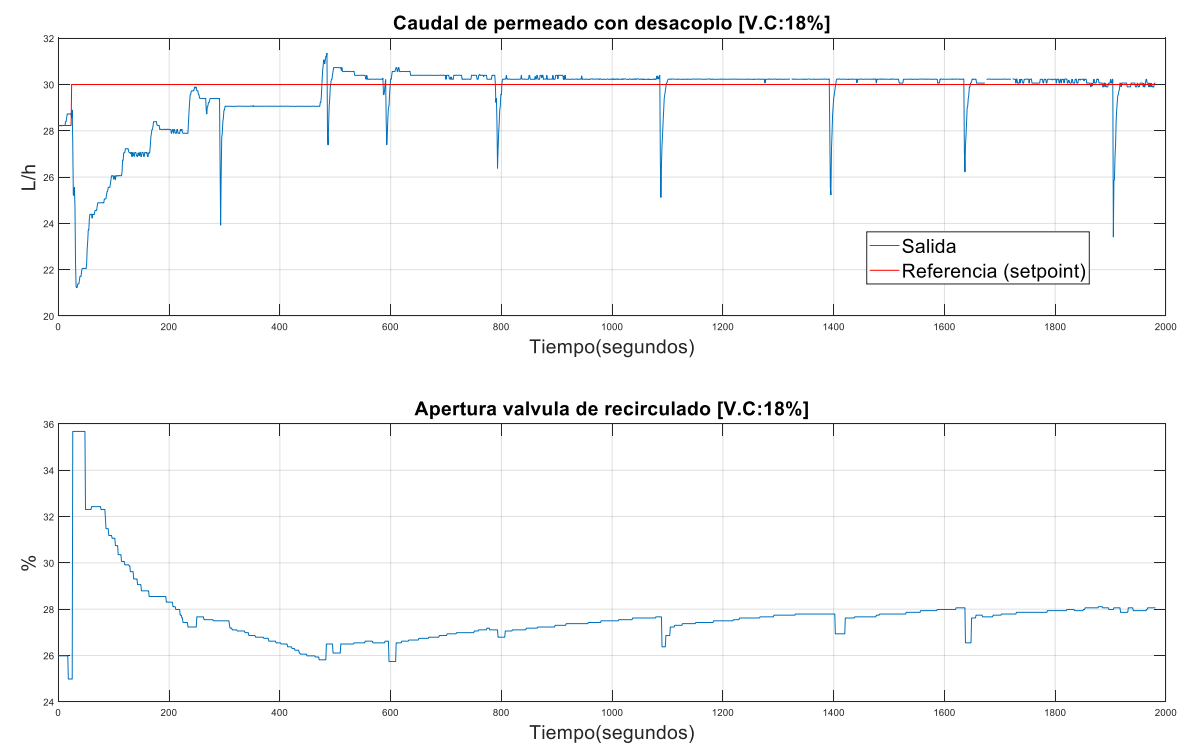

Figura 8: Comportamiento de presión y caudal con desacoplo (cont.).

\section{CONCLUSIONES Y FUTUROS TRABAJOS}

Después de experiencias preliminares con una planta piloto de nanofiltración para la reutilización de aguas residuales en agricultura, que se gestionaba de forma manual, se ha probado que el análisis y modelado de la misma, desde un punto de vista de la ingeniería de sistemas, ayuda a un mejor conocimiento de la planta y a determinar sus puntos de operación e identificar las variables que más influyen en la dinámica del sistema. Además, con la gestión de la misma de forma automática se pueden llegar a alcanzar unas condiciones óptimas de funcionamiento en cuanto a producción, seguridad y mantenimiento de las membranas. De los resultados obtenidos se puede concluir que el uso de modelos lineales presenta un buen comportamiento frente a las variaciones de las variables de entrada en torno al punto de operación determinado y al tipo de agua seleccionado. Por otra parte, la estrategia de control multivariable basada en controladores PI con anti-windup y desacopladores propuesta alcanza los puntos de operación definidos cumpliendo las especificaciones establecidas en la definición del problema.

En cuanto a la gestión de la planta por parte de los operarios de la Plataforma Solar de Almería, se ha establecido una metodología de trabajo de forma que se puedan reajustar el sistema para cada tipo de agua a tratar, maximizando el caudal de permeado y manteniendo condiciones de presión en su operación. 
Este trabajo ha servido como estudio de referencia para el uso de la planta con diferentes tipos de agua a tratar (procedente de EDAR, agua de fertirrigación, red de abastecimiento, etc.) Se trata de un trabajo inicial en el que se continúa trabajando en:

- Mejora de la instrumentación de la planta para considerar perturbaciones y aumentar el margen de regulación. Por ejemplo, incluir un conductímetro en la entrada y así poder considerar esta variable como perturbación, atenuándola con un controlador anticipativo.

- Desarrollo de un modelo físico de la planta que incluya el comportamiento de las membranas a fin de obtener el punto óptimo de operación en diferentes condiciones de forma matemática.

- Probar estrategias de control que consideren aspectos económicos y de eficiencia energética.

\section{Agradecimientos}

Este trabajo ha sido financiado con los Proyectos R+D+i DPI2014-56364-C2-1-R y DPI2017-85007-R del Ministerio de Economía, Industria y Competitividad y Fondos FEDER. Además, se agradece a la PSA-CIEMAT que haya permitido la utilización de sus instalaciones.

\section{MODELING AND CONTROL OF A PILOT NANOFILTRATION PLANT APPLIED TO THE REUSE OF WASTEWATER IN AGRICULTURE}

\begin{abstract}
Nowadays, the use of wastewater treatment facilities is necessary due to the scarcity of quality water resources. Nanofiltration plants are becoming more and more interesting, since they require less energy than other systems. A manually operated nanofiltration plant is used at Plataforma Solar de Almeria, based on the operators' experience. This work is devoted to the analysis, modeling and control of this kind of plant, which has helped to both improve knowledge and to determine the optimal operating point and identify the variables that most influence the behavior of the system. Furthermore, an automatic control architecture is proposed, based on a multivariable controller with decoupling. It is possible to achieve ideal operating conditions in terms of production, safety and membranes maintenance, as can be seen in the results presented.
\end{abstract}

Keywords: Multivariable control, Wastewater treatment, Dynamic models

\section{Referencias}

[1] Abbas, A.; 2006; Model predictive control of a reverse osmosis desalination unit; Desalination; Vol. 194; no 1-3; pp. 268-280.

[2] Aström, K.J.; Hägglund, T.; 2009; Control PID avanzado; Ed. Pearson; Madrid; España; 488 pp.

[3] Directiva 2000/60/CE del Parlamento Europeo y del Consejo, de 23 de octubre de 2000, por la que se establece un marco comunitario de actuación en el ámbito de la política de aguas (Diario Oficial n L 327 de 22/12/2000 p. 0001 - 0073)

[4] Millares, S.; Oller, I.; Sánchez, J.; Malato, S.; 2015; Eliminación de micro-contaminantes mediante combinación de sistemas de membrana (nano-filtración) y procesos avanzados de oxidación; Ed. Ciemat; Madrid; España; 340 pp.

[5] Stoller, M.; Mendes, R. S.; 2017; Advanced control system for membrane processes based on the boundary flux model; Separation and Purification Technology; Vol.175; pp. 527-535.

[6] Van der Bruggen, B.; et al.; 2001; Application of nanofiltration for removal of pesticides, nitrate and hardness from ground water: rejection properties and economic evaluation; Journal of Membrane Science; Vol.193; nº 2; pp. 239-248.

Anexo 1. Instrumentación de la planta piloto

\begin{tabular}{|l|l|}
\hline \multicolumn{1}{|c|}{ Símbolo } & \multicolumn{1}{|c|}{ Instrumento } \\
\hline FC1 & Controlador de caudal del lazo de control 1 \\
\hline FIT1A & $\begin{array}{l}\text { Transmisor indicador de caudal del lazo de } \\
\text { control 1 del concentrado }\end{array}$ \\
\hline FIT1B & $\begin{array}{l}\text { Transmisor indicador de caudal del lazo de } \\
\text { control 1 del recirculado }\end{array}$ \\
\hline FV1A & Válvula de control del caudal de concentrado \\
\hline FV1B & Válvula de control del caudal de recirculado \\
\hline PC2 & Controlador de presión del lazo de control 2 \\
\hline PIT2A & $\begin{array}{l}\text { Transmisor indicador de presión de entrada } \\
\text { del lazo de control 2 }\end{array}$ \\
\hline PIT2B & $\begin{array}{l}\text { Transmisor indicador de presión de salida del } \\
\text { lazo de control 2 }\end{array}$ \\
\hline FB2 & Bomba de control de la presión \\
\hline CIT0A & $\begin{array}{l}\text { Transmisor indicador de conductividad } \\
\text { eléctrica del concentrado }\end{array}$ \\
\hline CIT0B & $\begin{array}{l}\text { Transmisor indicador de conductividad } \\
\text { eléctrica del permeado }\end{array}$ \\
\hline TIT0 & $\begin{array}{l}\text { Transmisor indicador de temperatura del } \\
\text { recirculado }\end{array}$ \\
\hline FV0A & Válvula manual de la alimentación \\
\hline FV0B & Válvula manual de entrada a las membranas \\
\hline FV0C & Válvula manual de entrada a la membrana 1 \\
\hline FV0D & Válvula manual de entrada a la membrana 2 \\
\hline FV0E & Válvula manual de entrada a la membrana 3 \\
\hline FV0F & Válvula manual de salida a la membrana 1 \\
\hline FV0G & Válvula manual de salida a la membrana 2 \\
\hline FV0H & Válvula manual de salida a la membrana 3 \\
\hline FV0I & $\begin{array}{l}\text { Válvula manual para configurar en serie o } \\
\text { paralelo las tres válvulas }\end{array}$ \\
\hline
\end{tabular}

